\title{
Más continuidades que rupturas: un estudio sobre la inserción laboral diferencial de mujeres y varones en Argentina entre 2003 y 2017
}

\author{
Actis Di Pasquale, Eugenio* \\ Savino, Julia Victoria* \\ *Universidad Nacional de Mar del Plata, Argentina \\ E-mail: edipasq@mdp.edu.ar
}

Recibido: 16 diciembre de 2019 Aprobado: 21 diciembre de 2019

\begin{abstract}
Resumen
El objetivo del presente trabajo es analizar la evolución de las brechas de género entre la población ocupada de Argentina durante gran parte de las últimas dos década, habida cuenta del aparente estancamiento de la participación laboral femenina. Es decir, por un lado, determinar los cambios en las tasas de actividad, empleo y desocupación específicas. Y por otro lado, responder a la pregunta si se ha generado una disminución de las desigualdades en todas las dimensiones del trabajo, o bien, se siguen repitiendo los mismos mecanismos de discriminación, segregación vertical y horizontal que históricamente condicionaron la participación y bienestar de las mujeres. Las tendencias del mercado laboral se abordan a partir del análisis de las variaciones absolutas y relativas de distintos indicadores calculados para mujeres y varones, y de las brechas que surgen de los mismos. Asimismo se realizan análisis de descomposición de las variaciones en las tasas de empleo y del Índice de Disimilitud de Duncan. La fuente de datos es la Encuesta Permanente de Hogares (EPH) para el período 2003-2017. Los resultados obtenidos servirán para la descripción y diagnóstico de la situación y comportamiento diferencial de mujeres y varones en el mundo del trabajo. Asimismo, para la determinación de posibles cursos de acción que puedan generar políticas públicas cuya finalidad sea la igualdad y equidad entre mujeres y varones.
\end{abstract}

Palabras clave: Género, Mercado de trabajo, Segregación ocupacional

REVISTA DE INVESTIGACIÓN SIGMA / Vol. 06, Nº 2, 2019 (pág. 7-24) 


\title{
More continuities than ruptures: a study on the differential labor insertion of women and men in Argentina between 2003 and 2017.
}

\begin{abstract}
The objective of this paper is to analyze the evolution of gender gaps among the employed population of Argentina during much of the last two decades, given the apparent stagnation of female labor participation. That is, on the one hand, to determine the changes in specific activity, employment and unemployment rates. And on the other hand, answering the question if there has been a decrease in inequalities in all dimensions of work, or else the same mechanisms of discrimination, vertical and horizontal segregation that historically conditioned the participation and welfare of women. Labor market trends are addressed from the analysis of the absolute and relative variations of different indicators calculated for women and men, and the gaps that arise from them. Likewise, decomposition analyzes of variations in employment rates and the Duncan Disability Index are performed. The data source is the Permanent Household Survey (EPH) for the period 2003-2017. The results obtained will serve to describe and diagnose the situation and differential behavior of women and men in the world of work. Likewise, for the determination of possible courses of action that may generate public policies whose purpose is equality and equity between women and men.
\end{abstract}

Keywords: Gender, Labor market, Occupational segregation

\section{Introducción}

En los últimos treinta años la participación femenina en el mercado de trabajo latinoamericano ha experimentado un significativo dinamismo, pasando de un fuerte crecimiento en los '90 a una desaceleración a partir de los 2000 (Gasparini y Marchionni, 2015).

En este contexto, el mercado laboral de Argentina ha tenido un comportamiento similar, aunque con algunas excepciones. Si nos remitimos a la década de 1990, el fuerte crecimiento estuvo asociado fundamentalmente con las denominadas estrategias de supervivencia, es decir, frente al aumento del desempleo y/o el deterioro de los salarios reales del jefe varón se produjo la incorporación de más miembros del hogar al mercado de trabajo (Cerrutti, 2000; Castillo, Esquivel, Rojo, Tumini, y Yoguel, 2008). De acuerdo a Beccaria, Mauricio y Vázquez (2017) ese crecimiento llegó a ser a valores superiores a los del promedio de América Latina.
Asimismo, se profundizó la inserción ocupacional diferencial entre mujeres y varones y al interior de cada grupo. De acuerdo a Cortés (2003), en las ramas que se creó empleo masculino -construcción, transporte, y servicios personales- se concentraron los nuevos empleos de corto plazo, precarios y con bajos salarios. Por su parte, el $50 \%$ de las mujeres continuaron empleadas en ramas como la administración pública y los servicios sociales, donde la precariedad era menor, y el salario superior al promedio de las asalariadas mujeres. Pero en cambio, aquellas que estaban asalariadas en el servicio doméstico continuaron con condiciones contractuales precarias, debido a que alrededor del $90 \%$ eran trabajadoras no registradas.

No obstante, a partir de los primeros años de la década del 2000 se produjo un estancamiento en la participación femenina, situación que fue matizada con algunos años de descenso (Beccaria et al., 2017). Esta evolución se diferencia del resto de los países latinoamericanos, que tal 
como mencionamos, continuó aumentando a una tasa menor que la década previa. Y respecto a la inserción ocupacional, a partir del año 2003 y hasta el año 2006 cuando se detuvo el proceso de desindustrialización del empleo, también permaneció constante el proceso de feminización agregado. Asimismo, no se registraron cambios sustantivos en la tasa de feminidad de cada una de las ramas de actividad ni en la estructura ocupacional, por ende, el nivel de segregación permaneció constante (Castillo et al., 2008).

Algunos estudios realizados en los últimos años muestran la evolución diferencial de la inserción laboral de las mujeres y varones entre 2003 y 2011 (Actis Di Pasquale y Lanari, 2015) o bien hacen énfasis en la situación coyuntural entre 2016 y 2017 (MTEySS, 2017, 2018; Vozzi y Lafuente Duarte, 2017). En este sentido, surgen una serie de interrogantes sobre lo ocurrido en los últimos quince años con las brechas de género habida cuenta del estancamiento/disminución de la participación laboral femenina. ¿Cómo evolucionaron la cantidad y la calidad del empleo de mujeres y varones? ¿Hubo algún cambio en cada grupo de edad y por nivel educativo? Si sucedieron, ¿cómo afectaron a las variaciones en la participación y el empleo? Y la segregación histórica (vertical y horizontal) ¿se mantiene? ¿O bien la concentración femenina ha disminuido?

En virtud de ello, el objetivo del presente trabajo es analizar la evolución de las brechas de género entre la población ocupada entre 2003 y 2017 frente a la participación diferencial que se ha dado en las últimas décadas. La fuente de datos es la Encuesta Permanente de Hogares (EPH) y centramos el estudio en los cuartos trimestres y en la población de 15 a 64 años, dado que los indicadores calculados para este grupo resultan son más sensibles a los cambios que si utilizáramos al total de población.

El trabajo lo dividimos en apartados. En el segundo, presentamos la metodología a utilizar. En el tercero, analizamos la evolución de las tasas básicas de mercado de trabajo y por sexo. En el cuarto, efectuamos una descomposición sencilla de los cambios en las tasas de actividad y empleo de acuerdo a grupos etarios y nivel educativo. En el quinto, analizamos la empleabilidad diferencial de mujeres y varones, para dar cuenta de la calidad del empleo y la segregación ocupacional. Finalmente, en el sexto apartado se expone la reflexión final.

\section{Metodología}

En el presente estudio se utilizan herramientas de estadística descriptiva para el análisis de la evolución de los diferentes indicadores de mercado de trabajo, tales como las tasas básicas y otras complementarias. Asimismo, para determinar la descomposición de ciertas magnitudes se trabajaron con técnicas específicas.

Por un lado, efectuamos una descomposición simple de los cambios en la tasa de empleo de mujeres y varones respecto a la distribución de algunas variables (edad y educación), siguiendo la metodología empleada por Elías \& Ñopo (2010) como también por Gasparini, Marchionni, Badaracco, y Serrano (2015). Esto nos permite indagar si las modificaciones en la tasa de empleo obedecen a cambios en el nivel de ocupación al interior de cada grupo (efecto interno) y/o a variaciones en la distribución de la población respecto a los 
grupos conformados por esas variables (efecto composición).

Analíticamente:

$$
\Delta T E_{t}=\sum_{\nu_{L}} \bar{w}_{\ell_{k}} \Delta T E_{\ell_{k}}+\sum_{l_{L}} T E_{\ell_{k}} \Delta w_{\ell_{k}}
$$

Donde $\bar{w}_{k}=\left(w_{k t}+w_{k t+1}\right) / 2$; $\overline{T E}_{k}=\left(T E_{k t}+T E_{k t+1}\right) / 2 ; T E$ es la tasa de empleo definida como la proporción de población ocupada sobre la población total, del grupo $k$ en el tiempo $t ; \mathrm{y} w$ es la fracción de mujeres (o varones) en el grupo $k$ en el tiempo $t$.

De esta manera, el primer miembro de corresponder al efecto interno, dado que es un promedio ponderado de los cambios en la tasa de empleo de mujeres (o varones) dentro de cada grupo, y el segundo miembro al efecto composición, calculado como un promedio ponderado de los cambios en la distribución de mujeres (o varones) entre los grupos (composición).

Por otro lado, para calcular el nivel de segregación ocupacional horizontal utilizamos el Índice de Disimilitud de Duncan y Duncan (1955), que se define como:

$$
I D=\frac{\sum_{i}\left|v_{i}-m_{i}\right|}{2}
$$

Donde $v_{i}$ es el porcentaje de varones trabajando en la rama de actividad $i$ y $m_{i}$ es el porcentaje de mujeres trabajando en la rama de actividad $i$. Este índice varía entre cero y uno. Toma el valor cero cuando la distribución ocupacional de varones y mujeres es idéntica, y uno, cuando varones y mujeres no se superponen en ninguna ocupación, es decir que existe una segregación ocupacional total. Este índice suele interpretarse como la proporción de mujeres (o varones) ocupadas que sería necesario cambiar de ocupación para lograr la perfecta integración.

De acuerdo a Amarante y Espino (2002) una de las limitaciones del ID es que sus variaciones pueden deberse tanto a los cambios en la estructura ocupacional de la fuerza de trabajo como a los cambios en la composición por sexo de las ocupaciones. Para aislar estos dos efectos suele descomponerse la variación del ID en el componente que refleja la modificación estructural en las ocupaciones (efecto ocupación) $y$ en el que muestra las variaciones en la composición por sexo de las ocupaciones (efecto composición).

Para ello se debe considerar el ID para dos momentos en el tiempo, tomando como base el del año inicial. Al ID del último año se lo transforma de la siguiente manera:

Para calcular el efecto ocupación, se consideran los porcentajes de varones y mujeres del año base con la estructura ocupacional del año final. De esta manera, se simulan los cambios en la estructura ocupacional dejando constante la composición por sexo en las ramas de actividad.

Ocup $=\frac{1}{2}\left[\sum_{i}\left|\frac{v_{i 1} * T_{i 2}}{\sum_{i} v_{i 1} * T_{i 2}}-\frac{m_{i 1} * T_{i 2}}{\sum_{i} m_{i 1} * T_{i 2}}\right|-\sum_{i}\left|\frac{v_{i 1} * T_{i 1}}{\sum_{i} v_{i 1} * T_{i 1}}-\frac{m_{i 1} * T_{i 1}}{\sum_{i} m_{i 1} * T_{i 1}}\right|\right]$

Para calcular el efecto composición, se consideran los porcentajes de varones y mujeres del año final con la estructura ocupacional del año base. De esta manera, se simulan los cambios en la composición por sexo en las ramas de actividad dejando constante la estructura ocupacional.

$$
\begin{aligned}
& \text { Comp }=\frac{1}{2}\left[\sum_{i}\left|\frac{v_{i 2} * T_{i 1}}{\sum_{i} v_{i 2} * T_{i 1}}-\frac{m_{i 2} * T_{i 1}}{\sum_{i} m_{i 2} * T_{i 1}}\right|-\sum_{i}\left|\frac{v_{i 1} * T_{i 1}}{\sum_{i} v_{i 1} * T_{i 1}}-\frac{m_{i 1} * T_{i 1}}{\sum_{i} m_{i 1} * T_{i 1}}\right|\right] \\
& v_{i t} \text { es el porcentaje de varones en la } \\
& \text { ocupación } i \text { en el momento } t, m_{i t} \text { es el porcentaje }
\end{aligned}
$$


de mujeres en la ocupación $i$ en el momento $t$ y $T_{i t}$ es el número total de trabajadores en la ocupación $i$ en el momento $t$.

Sin embargo, esta descomposición tiene algunas limitaciones. Por un lado, la variación total del ID resulta ser la suma del efecto ocupación y composición más un término residual que mide la interacción entre ambos, tal como lo calculamos en este capítulo.

$$
\Delta I D=\text { Ocup }+ \text { Comp }+ \text { Residual }
$$

Por otro lado, se presenta un problema de número índice, ya que los resultados serán diferentes según el año base que se considere.

Para solucionar, ambos inconvenientes en la literatura se sugiere considerar un promedio de los valores del año inicial y final (Flückiger y Silber, 1999). No obstante, optamos por realizar el cálculo original, ya que capta la complejidad de tratar de escindir estos fenómenos al trabajar en términos cronológicos. De esta manera, los resultados tendrían un grado de aproximación mayor a la realidad.

\section{La evolución diferencial de las tasas básicas de mercado de trabajo}

Previo al estudio de los cambios que se produjeron en las tasas básicas (actividad, empleo, desocupación y subocupación), realizamos una periodización de la evolución del Producto Bruto Interno (PBI), teniendo en cuenta que la evolución del nivel de actividad económica es uno de los principales factores que puede impactar en su dinámica. De esta manera se pueden distinguir dos etapas diferenciadas: a) crecimiento significativo (2003-2011): con tasas de variación del PBI elevadas los primeros años (cercanas al 10\%), disminución-recuperación durante la crisis 2008/2009 y desaceleración sobre el final; b) estancamiento (2012-2017): solo un leve aumento entre puntas, pero con fuertes oscilaciones interanuales.

En este contexto, durante la primera etapa, la tasa de actividad total tuvo un comportamiento descendente, pasando del 69,3\% al 67,7\%, mientras que la tasa de empleo total creció en casi 4 puntos porcentuales (p. p.) con una menor participación de subocupados. Es decir, que el sostenido crecimiento económico acontecido en esos años generó una mayor empleabilidad y la razón principal de la reducción de la participación laboral fue la significativa caída en la cantidad de desocupados (Figura 1).

$\mathrm{Si}$ analizamos los comportamientos diferenciales entre sexos en el mismo período, encontramos que la tasa de actividad de los varones disminuyó en una menor proporción respecto a la de las mujeres $(-1,0$ p. p. frente a $-2,0$ p. p., respectivamente). Asimismo, los aumentos del empleo fueron del 4,6 p. p. y 3,2 p. p. respectivamente. No obstante, el nivel de empleo conseguido por las mujeres en 2011 (apenas superior al 50\%) ya lo habían logrado en 2005, mientras que, el alcanzado por los varones al final de la etapa resulta ser el mayor valor histórico hasta ese momento $(76,5 \%)$. Se puede afirmar que el nivel de ocupación de los varones fue procíclico durante toda la etapa y el de las mujeres tuvo un estancamiento con una respuesta contracíclica en 2008.

En la segunda etapa, las variaciones en los indicadores acontecidas a lo largo de los siete años no permiten inferir una clara tendencia. 
Si bien hay una aparente estabilidad de la participación $\boldsymbol{y}$ el empleo al comparar 2011 con 2017, en el interior de esta etapa se vislumbran los vaivenes de los ciclos económicos y del cambio en el rumbo de la política económica a partir de 2016.

Las tasas de actividad y de empleo para el total de la población, disminuyen entre 2011 y 2014 y se recupera en 2016-2017, llegando a un valor similar que al inicio de la etapa. Estas variaciones de los primeros años se encuentran explicadas por descensos en la participación y empleo de los varones, que no pudieron ser contrarrestadas por las leves mejoras para las mujeres. Recién en los dos últimos años, la mayor cantidad de trabajadoras logra compensar las mermas en el empleo masculino, con lo cual, la segunda etapa termina con una similar tasa de empleo total (-0,2 p. p.), pero con una disminución para los varones $(-2,1$ p. p.) y un aumento para las mujeres $(+1,7$ p. p.). Cabe aclarar que una mejora en la tasa de empleo no significa que se haya producido una creación genuina de puestos de trabajo, aspecto que profundizaremos en el quinto apartado.

Por su parte, las tasas de desocupación y subocupación registran un leve incremento en el período. Esas variaciones se encuentran explicadas tanto por el comportamiento de mujeres como de varones, pero principalmente por el de ellos.

En definitiva, la tasa de actividad de mujeres tuvo un descenso entre 2003-2011 y luego una recuperación entre 2011-2017, con lo cual su valor se mantuvo casi constante $(+0,1 \mathrm{p}$. p.). En cambio en los varones, fue descendiendo paulatinamente en cada uno de esos años $(-2,7 \mathrm{p}$. p. entre puntas). Por su parte, la tasa de empleo de mujeres tuvo una tendencia de aumento $(+5$ p. p.) y en el caso de los varones aumentó entre 2003 y 2011 y disminuyó sobre el final, quedando en un valor por encima que el inicio de la serie $(+2,5$ p. p.). Asimismo, disminuyen la desocupación y subocupación de mujeres en mayor proporción que la de varones. Estos cambios dan cuenta que la comparación entre los extremos de la serie (2003 y 2017) arrojó disminuciones en las brechas de las cuatro tasas básicas, siendo el aumento diferencial del empleo de mujeres respecto a varones la variable que reviste mayor relevancia. 
Tasa de Actividad

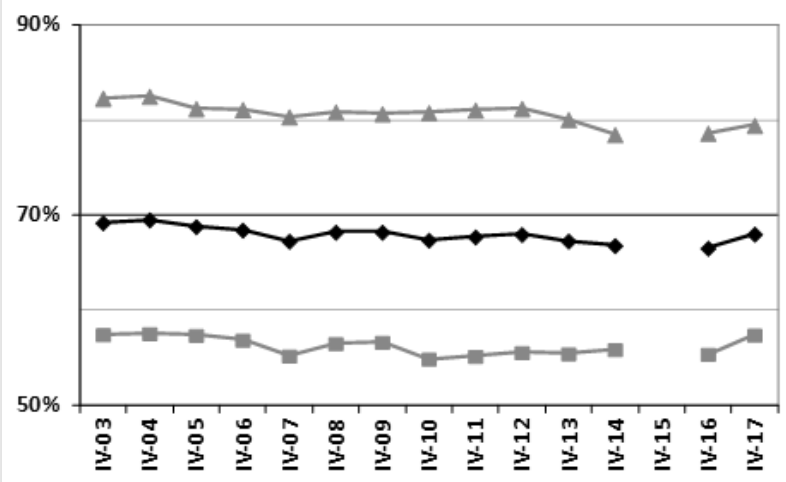

Tasa de Subocupación

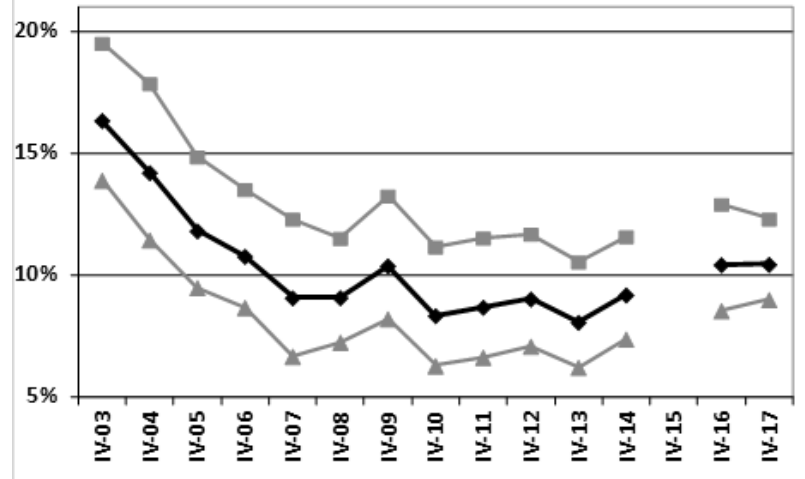

Tasa de Empleo

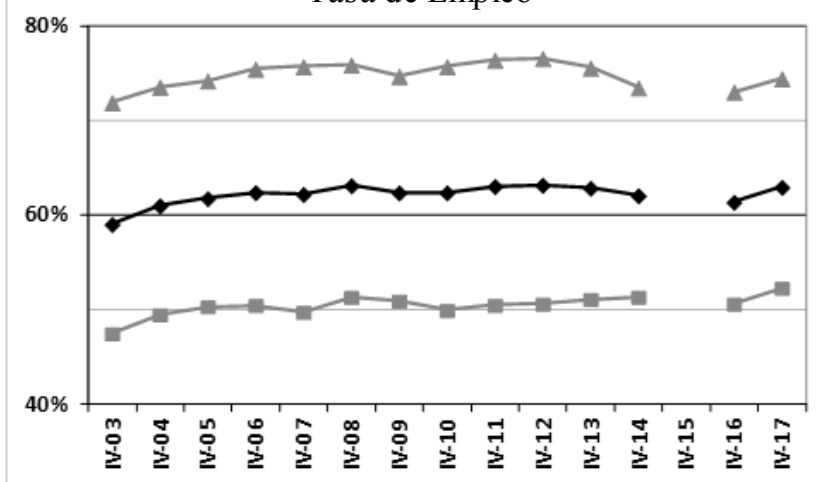

Tasa de Desocupación

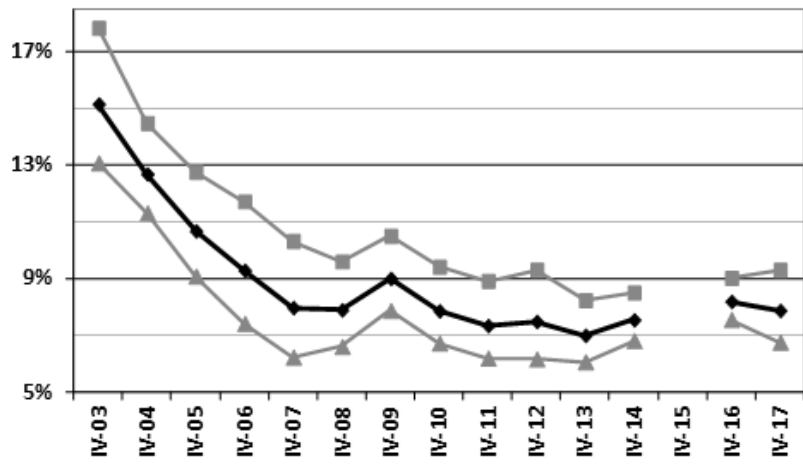

$\sim$ Total

Mujeres

- Varones

Figura 1: Tasas Básicas del mercado de trabajo (población de 15 a 65 años). Total aglomerados urbanos (IV Trimestre 2003 - IV trimestre 2017)

Fuente: Elaboración propia en base a microdatos EPH.

En este sentido, surgen algunos interrogantes en torno a ese incremento del nivel de ocupación. Por un lado, las modificaciones en el empleo, ¿se dieron en todos los grupos de edad? ¿Y por nivel educativo? Y esos cambios, ¿se deben al nivel de ocupación al interior de cada grupo (efecto interno) y/o a variaciones en la distribución de la población dentro de los grupos? Por otro lado, la mejora del empleo femenino que fue superior al de los varones, ¿implica que se insertaron en empleos de calidad? ¿En qué ramas de actividad se incorporaron? ¿Cómo se vio afectada la segregación ocupacional? En los próximos nos dedicamos a responder estos interrogantes, enfocándonos en las variaciones de la tasa de empleo en 2003, 2011 y 2017.

\section{Descomposición de la tasa específica de empleo por grupos de edad $y$ nivel educativo}

Como estudiamos en el apartado anterior, la tasa de empleo de los varones aumentó entre 2003 y 2011 y luego disminuyó entre 2011 y 2017. En cambio, la tasa de empleo de las mujeres tuvo incrementos en ambas etapas. Esas variaciones pueden estar provocados por diversos factores extraeconómicos, tales como demográficos, sociales y culturales. De todas las posibles variables que integran esas dimensiones, a continuación analizaremos solamente dos: 
la composición por grupos de edad, porque es una variable que da cuenta de la permanencia en el mercado a lo largo del ciclo vital, y el nivel educativo debido a que, como demuestran Gasparini et al (2015), las mejoras en educación son las que explican en mayor proporción el aumento de la participación de las mujeres en América Latina en los 2000.

Respecto a la tasa de empleo por grupos de edad, encontramos que en el caso de los varones esas variaciones se dieron en una magnitud similar en casi todos los grupos de edad. Y en el caso de las mujeres esos cambios estarían explicados por el crecimiento del empleo de las mayores de 25 años, con especial énfasis de las mayores de 45 años durante la segunda etapa. Si bien se redujo la TE de las más jóvenes, ello no logró contrarrestar el mencionado aumento (Figura 2).

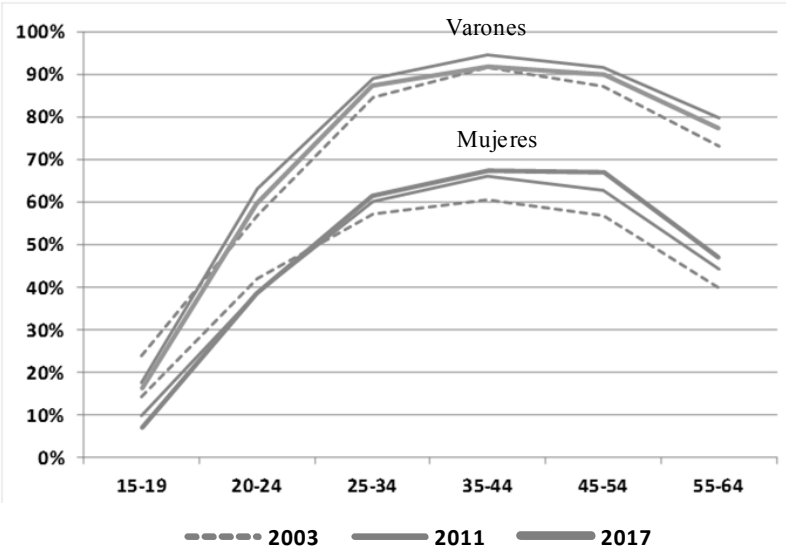

Figura 2: Tasa de empleo por grupos de edad y sexo (población de 15 a 65 años). Total aglomerados urbanos (cuartos trimestres de 2003, 2011 y 2017)

Fuente: Elaboración propia en base a microdatos EPH.

Sin embargo, en estos quince años se produjo un envejecimiento de la población, lo cual podría estar afectando los valores que alcanzan estas tasas. Según estimaciones de la $\mathrm{EPH}$, en 2003 la población de 35 y más años representaba el 48,7\% y en 2017 llegó al 53,1\%. En este sentido, el análisis de descomposición de la tasa de empleo intenta separar la parte que se debe a modificaciones en el nivel de ocupación al interior de cada grupo (efecto interno) de la fracción que se debe a variaciones en la distribución de la población por grupos de edad (efecto composición).

Los resultados indican que tanto para varones como para mujeres los cambios en cada etapa se encuentran explicados principalmente por el efecto interno. Es decir, que si no se hubieran producido cambios en la estructura etaria en esa década, la tasa de empleo hubiera variado en el mismo sentido. No obstante, en el caso de los varones el efecto composición positivo en la primera etapa permitió explicar más de la mitad del aumento entre puntas (es decir, por haber una mayor participación en los grupos etarios más altos), mientras que en el caso de las mujeres, este efecto fue menor (Tabla 1). 


\section{Tabla 1}

Descomposición de los cambios en las tasas de empleo especificas por grupos de edad (población de 15 a 65 años). Total aglomerados urbanos (cuartos trimestres de 2003, 2011 y 2017).

\begin{tabular}{|r|c|c|c|c|c|c|}
\hline \multirow{2}{*}{ TASA DE EMPLEO } & \multicolumn{3}{|c|}{ VARONES } & \multicolumn{3}{c|}{ MUJERES } \\
& $\mathbf{2 0 0 3 - 2 0 1 1}$ & $\mathbf{2 0 1 1 - 2 0 1 7}$ & $\mathbf{2 0 0 3 - 2 0 1 7}$ & $\mathbf{2 0 0 3 - 2 0 1 1}$ & $\mathbf{2 0 1 1 - 2 0 1 7}$ & $\mathbf{2 0 0 3 - 2 0 1 7}$ \\
\hline Diferencia observada & \multicolumn{1}{|c|}{} & $\mathbf{- 2 . 1}$ & $\mathbf{2 . 5}$ & 3.2 & $\mathbf{1 . 8}$ & $\mathbf{5 . 0}$ \\
\hline Efectos & & & & & & \\
\hline Interno & 3.3 & -2.2 & 1.1 & 2.5 & 1.3 & 3.9 \\
\hline Composición & 1.3 & 0.1 & 1.4 & 0.7 & 0.4 & 1.1 \\
\hline
\end{tabular}

Fuente: Elaboración propia en base a microdatos EPH.

La otra variable que utilizamos es el nivel educativo. En el caso de los varones, la tasa de empleo por nivel educativo da cuenta que la única medida que disminuye en las dos etapa es la que corresponde al nivel bajo. La tasa calculada para el resto de los niveles tiene el mismo comportamiento que el agregado: aumento y disminución. Y entre las mujeres, la tasa de empleo de niveles bajo e intermedio disminuye año tras año y aumenta en los niveles educativos medios y altos (Figura 3).

Al igual que lo analizado por grupos de edad, hay que tener presente la distribución de esos grupos. En este sentido, durante el período estudiado, el nivel de formación de toda la población ocupada aumentó en forma significativa. De hecho, el porcentaje de población ocupada con nivel educativo bajo e intermedio ha disminuido ( $-48 \%$ y $-26 \%$, respectivamente) y como contrapartida, la proporción con nivel medio y alto ha aumentado $(+25 \% \mathrm{y}+34 \%)$. De esta manera, las mujeres continúan alcanzando un mayor nivel de capital humano que los varones: entre el $31 \%$ de las mujeres posee estudios universitarios completos, frente al $17 \%$ de los varones.

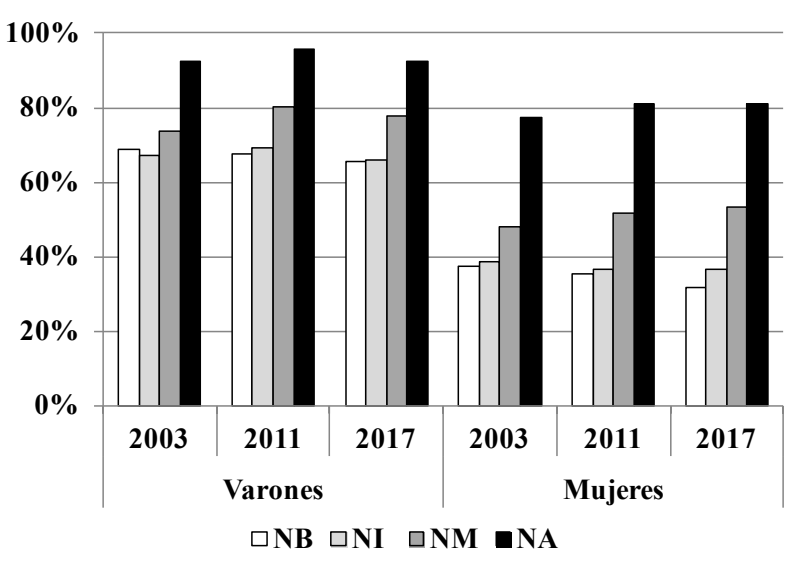

Figura 3: Tasas de empleo específicas por nivel educativo y sexo (población de 15 a 65 años). Total aglomerados urbanos (cuartos trimestres de 2003, 2011 y 2017)

Fuente: Elaboración propia en base a microdatos EPH.

Nuevamente,elanálisisdedescomposición de la tasa de empleo nos permitirá distinguir el efecto interno del efecto composición. En el caso de los varones, prevalece el efecto interno en cada etapa. No obstante, durante la segunda etapa, la mayor educación fue lo que permitió morigerar la caída de 2,1 p.p., dado que el efecto composición fue de $+0,7$ p.p. Y de hecho, si analizamos entre puntas, es el efecto composición es el que explica las dos terceras partes de esos cambios.

Por su parte, entre las mujeres, la variación en la tasa de empleo se encuentra explicada 
principalmente por el mayor nivel educativo alcanzado por las mujeres tanto en cada etapa como entre puntas (efecto composición de $+3,5$ p.p.), el cual fue ampliamente superior al de los varones (Tabla 2).

\section{Tabla 2}

Descomposición de los cambios en las tasas de empleo especificas por nivel educativo (población de 15 a 65 años). Total aglomerados urbanos (cuartos trimestres de 2003, 2011 y 2017).

\begin{tabular}{|r|c|c|c|c|c|c|}
\hline \multirow{2}{*}{ TASA DE EMPLEO } & \multicolumn{3}{|c|}{ VARONES } & \multicolumn{3}{c|}{ MUJERES } \\
& $\mathbf{2 0 0 3 - 2 0 1 1}$ & $\mathbf{2 0 1 1 - 2 0 1 7}$ & $\mathbf{2 0 0 3 - 2 0 1 7}$ & $\mathbf{2 0 0 3 - 2 0 1 1}$ & $\mathbf{2 0 1 1 - 2 0 1 7}$ & $\mathbf{2 0 0 3 - 2 0 1 7}$ \\
\hline Diferencia observada & 4.6 & -2.1 & 2.5 & 3.2 & 1.8 & 5.0 \\
\hline Efectos & & & & & & \\
\hline Interno & 3.6 & -2.8 & 0.8 & 1.1 & 0.4 & 1.5 \\
\hline Composición & 1.1 & 0.7 & 1.8 & 2.1 & 1.3 & 3.5 \\
\hline
\end{tabular}

Fuente: Elaboración propia en base a microdatos EPH.

Nota: las diferencias en las sumatorias de los efectos con respecto al total se deben al redondeo de cifras.

En resumen, al descomponer los cambios ocurridos en cada etapa podemos afirmar que: i) en el caso de los varones, las variaciones en cada etapa obedecen fundamentalmente a un efecto interno, con lo cual otras variables pueden haber sido los determinantes responsables de ese comportamiento. Sin embargo, en los cambios 2003-2017, la educación aparece como la responsable principal del aumento; y ii) en el caso de las mujeres, las variaciones se deben eminentemente al mayor nivel de educación formal.

Cabría indagar si esta mejora en la cantidad de empleo para las mujeres ha ido acompañada de una mejor calidad en los puestos de trabajo, menor segregación ocupacional y una reducción de la brecha salarial.

\section{Acerca de la empleabilidad diferencial de mujeres y varones}

Este último apartado lo dividimos en tres partes: en primer lugar, un análisis de la evolución de las categorías ocupacionales y del trabajo no registrado; en segundo lugar, analizamos los factores asociados a la brecha de ingresos; y en tercer lugar, una descomposición de los cambios en la segregación ocupacional.

\section{La calidad de los puestos de trabajo}

La composición del empleo, en base a la categoría ocupacional y la tasa de registración, permite inferir acerca del acceso que tienen los trabajadores y las trabajadoras a los principales derechos laborales. En este sentido, en la primera etapa se presenta un marcado aumento de la población asalariada, alcanzando en 2011 máximos históricos para mujeres (pasó del 79,8\% al $82,7 \%$ ) y varones (de $71,8 \%$ a $75,3 \%$ ). Este crecimiento fue preferentemente en puestos de trabajo registrados, habida cuenta del significativo descenso de la proporción de asalariadas y asalariados no registrados. Asimismo, como contrapartida se dio un sostenido descenso de la población por cuenta propia, llegando a mínimos 
históricos de 13,8\% y 19,3\%, respectivamente.

En la segunda etapa, caen los porcentajes de población asalariada hasta el 79,8\% y $73,6 \%$, respectivamente, es decir, que vuelven a niveles iguales o similares a los de 2003 . Como contrapartida aumenta la proporción de cuentapropismo llegando a máximos históricos en el caso de las mujeres $(17,3 \%)$ y cercanos a ello en los varones (21,9\%) . Cabe destacar que este deterioro se dio con mayor intensidad entre 2016 y 2017. Por su parte, el trabajo no registrado se mantuvo en

Porcentaje de población asalariada y por cuenta propia

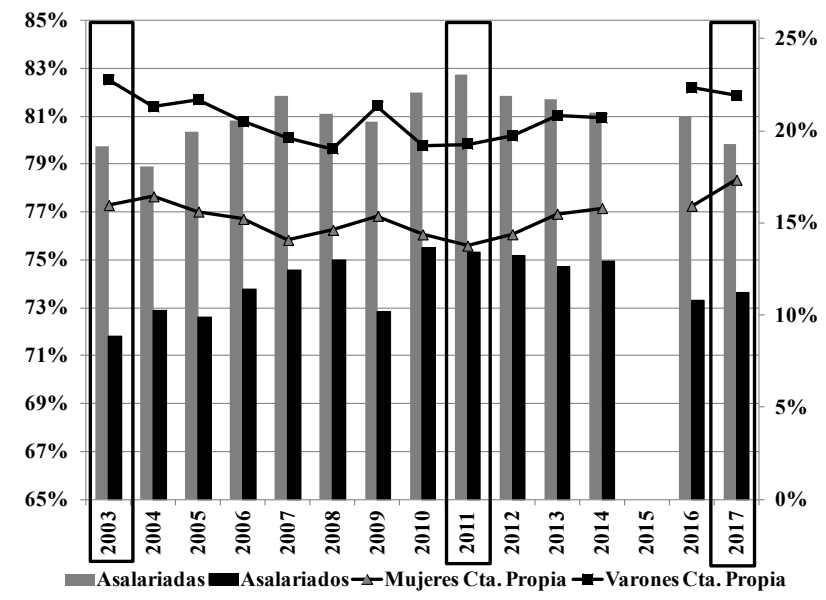

niveles similares durante toda la segunda etapa, con una menor brecha entre sexos que al inicio (Figura 4).

En definitiva, tanto la desaceleración de la tasa de empleo de mujeres como la disminución en el caso de los varones, ocultan un deterioro en la relación laboral, debido a nueva composición del empleo. Es decir, que el aumento de la cantidad de mujeres ocupadas fue preferentemente como cuentapropistas; y la caída del empleo de varones, fue originada principalmente por puestos que eran en relación de dependencia.

Proporción de población asalariada no registrada

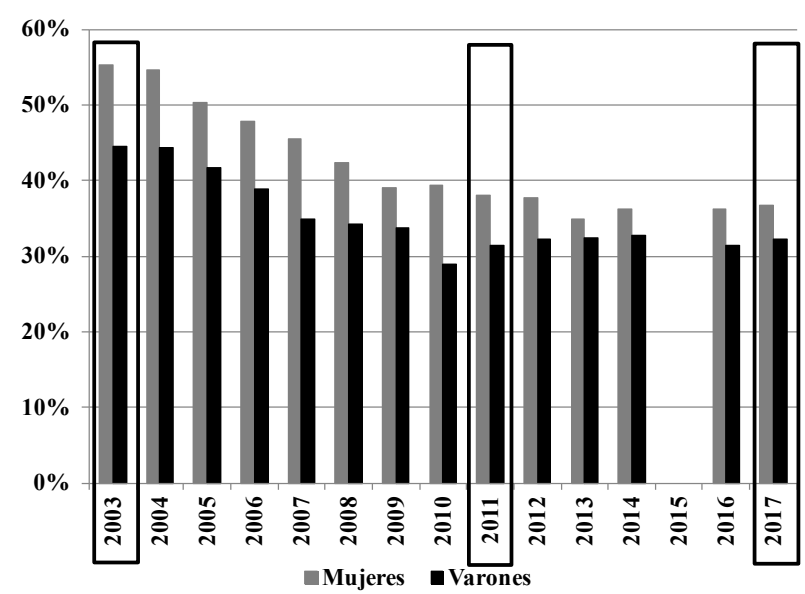

Figura 4: Categoría ocupacional y registración laboral de mujeres y varones (población de 15 a 65 años). Total aglomerados urbanos (cuartos trimestres de 2003, 2011 y 2017)

Fuente: Elaboración propia en base a microdatos EPH.

Segregación ocupacional y horas de trabajo remunerado como determinantes de la brecha de ingresos

Entre 2003 y 2008 la brecha salarial en Argentina se ubicó en promedio por encima del 30\% inclusive, y a partir de allí, los valores estuvieron en torno al 25\% (Actis Di Pasquale y Lanari, 2015), hasta el 2017 inclusive. En los años seleccionados para este análisis, los salarios de las mujeres crecen en promedio a una tasa más alta que la de los varones, con lo cual la brecha pasa del $31 \%$ al $26,1 \%$ y finaliza en $24,3 \%$ (Tabla $3)$.

El hecho que se presente una brecha salarial mensual en contra de las mujeres, estaría demostrando la existencia de una división sexual del trabajo remunerado y no remunerado, la existencia de diferencias en las 
jerarquías laborales y la concentración femenina en ciertas ramas de actividad. En este sentido, se puede indagar sobre la evolución de algunos indicadores que den cuenta estos factores,

\section{Tabla 3}

Indicadores de brecha de ingresos, horas de trabajo remunerado y segregación ocupacional (población de 15 a 65 años). Total aglomerados urbanos (cuartos trimestres de 2003, 2011 y 2017).

\begin{tabular}{|r|c|c|c|c|c|c|}
\hline INDICADORES DE GÉNERO & 2003 & 2011 & 2017 & $2003-2011$ & $2011-2017$ & $2003-2017$ \\
\hline Brecha de Ingresos & & & & & & \\
\hline Brecha relativa (\%) & 31.0 & 26.1 & 24.3 & $\downarrow$ & $\downarrow$ & $\downarrow$ \\
\hline $\begin{array}{r}\text { Horas semanales - Trabajo } \\
\text { rem. }\end{array}$ & & & & & \\
\hline Mujeres (hs) & 32.5 & 34.4 & 32.0 & $\uparrow$ & $\downarrow$ & $\downarrow$ \\
\hline Varones (hs) & 45.3 & 45.6 & 43.4 & $\uparrow$ & $\downarrow$ & $\downarrow$ \\
\hline Brecha Absoluta (hs) & 12.8 & 11.2 & 11.4 & $\downarrow$ & $\uparrow$ & $\downarrow$ \\
\hline Segregación vertical roujeres en puestos directivos \\
\hline $\begin{array}{r}\text { Mu) } \\
\text { Varones en puestos directivos }\end{array}$ & 4,6 & 4,9 & 4,8 & $\uparrow$ & $\downarrow$ & $\uparrow$ \\
\hline $\begin{array}{r}\text { Razón entre mujeres y varones } \\
\text { (\%) }\end{array}$ & 40.4 & 40.4 & 47.8 & $=$ & $\uparrow$ & $\downarrow$ \\
\hline Segregación horizontal & & & & & & $\downarrow$ \\
\hline Índice de feminización & 0,81 & 0,77 & 0,82 & $\downarrow$ & $\uparrow$ & $\uparrow$ \\
\hline Índice de Disimilitud de Duncan & 0,468 & 0,415 & 0,417 & $\downarrow$ & $=$ & $\downarrow$ \\
\hline
\end{tabular}

Fuente: Elaboración propia en base a microdatos EPH.

A continuación se resumen los principales resultados:

Respecto a las horas semanales, entre 2003 y 2017 se redujo la brecha absoluta debido fundamentalmente a la disminución de la cantidad de horas que dedican los hombres.

En cuanto a la segregación vertical, en la segunda etapa aumentó la razón entre mujeres y varones en puestos directivos públicos y privados (del 40,4\% al 47,8\%). Sin embargo, esto no necesariamente representó un avance para las mujeres, dado que el porcentaje de asalariadas en tales como las horas semanales dedicadas al trabajo remunerado, la segregación vertical y la segregación horizontal(Tabla 3). 
hasta 0,415$)$ para luego mantenerse en el mismo nivel (0,417 en 2017). Esto implicaría que los cambios más pronunciados para disminuir la segregación horizontal postcrisis 2002, se dieron en los primeros años cuando se registró un fuerte crecimiento del empleo (principalmente asalariado) que fue superior para los varones. Luego la mayor reducción relativa de asalariados varones parece no haber modificado el grado de asimetría de las ocupaciones.

Sin embargo, el ID puede variar por una modificación de la estructura ocupacional o bien, por cambios en la composición por sexo de las ocupaciones, tal como analizamos a continuación.

\section{Descomposición del Índice de Duncan y análisis por rama de actividad}

La variación del índice suele descomponerse en el componente que refleja las variaciones en la composición por sexo de las ocupaciones (efecto composición) y el que refleja la modificación estructural en las ocupaciones (efecto mixto). De acuerdo a Anker (1998), se recomienda tener en cuenta estos efectos para evitar sesgos interpretativos en los posibles cambios en el nivel de segregación.

Entre 2003 y 2011, casi el $85 \%$ de la disminución (o sea 0,045 de los 0,053 que cae el índice) se encuentra explicada por el efecto composición, siendo el efecto mixto casi nulo. El término residual, que mide la interacción entre efecto mixto y composición dio un valor relativamente bajo, que representa poco más del 15\%. En cambio, en la segunda etapa el índice no tiene cambios significativos (baja en 0,002) al igual que ambos efectos y el término residual, que son casi nulos (Tabla 4).

\section{Tabla 4}

Descomposición de la variación en el índice de Duncan (Asalariados) (población de 15 a 65 años). Total aglomerados urbanos (cuartos trimestres de 2003, 2011 y 2017).

\begin{tabular}{|r|c|c|c|}
\hline \multicolumn{1}{|c|}{ INDICE DE DUNCAN } & $\mathbf{2 0 0 3 - 2 0 1 1}$ & $\mathbf{2 0 1 1 - 2 0 1 7}$ & $\mathbf{2 0 0 3 - 2 0 1 7}$ \\
\hline Diferencia & -0.053 & 0.002 & -0.051 \\
\hline Efectos & & & \\
\hline Composición (sexo) & -0.045 & -0.002 & -0.050 \\
\hline Mixto (ocupación) & 0.001 & 0.003 & 0.004 \\
\hline Residual & -0.008 & 0.001 & -0.006 \\
\hline
\end{tabular}

Fuente: Elaboración propia en base a microdatos EPH.

Por ende, este análisis permite comprobar que lo que se produjo fue una mejora en la composición por sexo de las ramas de actividad, con una estructura de ocupación que permanece casi inalterada. No obstante, aún resta indagar si las ramas en que mejora la composición por sexo son las que tienen mayor proporción de mujeres o aquellas que ocupan preferentemente a varones.

Para ello, se seleccionaron las ramas de actividad basados en el criterio de Actis Di Pasquale y Lanari (2015), que incluye cada una de las que emplea en promedio más del 10\% de mujeres (Comercio; Administración 
Pública y Defensa; Salud Humana y Servicios Sociales; Enseñanza; Servicio Doméstico), más del 10\% de varones (Construcción; Comercio; Transporte, Almacenamiento y comunicación; Administración Pública y Defensa) y aquellas con un nivel de concentración similar de ambos sexos (Industrias Textiles, confecciones y calzado; Comercio; Restaurantes y Hoteles; Intermediación Financiera; Administración Pública y Defensa). Dado que algunas cumplen dos o tres de los requisitos, el total lo conforman diez ramas de actividad que nuclean al $78 \%$ del total de la población asalariada.

Al analizar los cambios entre 2003 y 2017, se observa que: a) las ramas de mayor proporción de varones (Construcción; Transporte, Almacenamiento y comunicación) continuaron consolidando esa concentración; b) de las que concentran similar proporción de mujeres y varones, Industrias Textiles, confecciones y calzado tuvo pérdidas de empleo de mujeres frente a un incremento equiproporcional de varones, razón por la cual se invirtió la relación de feminización (el índice pasó de 1,30 a 0,76). Por ende, si bien este cambio afecta a la baja el valor del índice de Duncan calculado con anterioridad, lo cierto es que impacta en la menor presencia relativa de mujeres en estos puestos de trabajo; c) el resto de las ramas que concentran similar proporción de ambos sexos (Comercio; Restaurantes y Hoteles; Intermediación Financiera; Administración Pública y Defensa) tuvieron un incremento porcentual superior de mujeres que de varones, por lo tanto, el índice de feminización aumentó (las ramas se hicieron más igualitarias); d) dos de las ramas femeninas (Educación; Salud Humana y Servicios Sociales) tuvieron aumentos proporcionalmente mayores de asalariados que de asalariadas, por ende, el índice de feminización disminuyó en ambas ramas; e) por último, servicio doméstico, está entre las tres ramas que más trabajo asalariado generaron en el conjunto de la economía (junto a Administración Pública y Educación), y representa la rama que más empleo generó entre las mujeres. En virtud de ello, la proporción dentro del total de asalariadas creció a valores cercanos al 22\% (Tabla 5). 
Tabla 5

Estructura ocupacional por sexo e índice de feminización. Ramas seleccionadas de población asalariada (población de 15 a 65 años). Total aglomerados urbanos (cuartos trimestres de 2003, 2011 y 2017)

\begin{tabular}{|c|c|c|c|c|c|c|c|c|c|}
\hline \multirow{2}{*}{ RAMA DE ACTIVIDAD } & \multicolumn{3}{|c|}{2003} & \multicolumn{3}{|c|}{2011} & \multicolumn{3}{|c|}{2017} \\
\hline & $\% \mathbf{M}$ & $\% \mathrm{~V}$ & IF & $\% \mathbf{M}$ & $\% \mathrm{~V}$ & IF & $\% \mathbf{M}$ & $\% \mathrm{~V}$ & IF \\
\hline Ind. Textiles, confecciones y calzado & $3.1 \%$ & $1.9 \%$ & 1.30 & $2.6 \%$ & $2.9 \%$ & 0.69 & $1.8 \%$ & $1.9 \%$ & 0.76 \\
\hline Construcción & $0.6 \%$ & $9.2 \%$ & 0.05 & $0.5 \%$ & $12.1 \%$ & 0.03 & $0.6 \%$ & $11.6 \%$ & 0.04 \\
\hline Comercio (por mayor y menor) & $10.6 \%$ & $14.8 \%$ & 0.58 & $11.9 \%$ & $14.3 \%$ & 0.64 & $10.8 \%$ & $14.1 \%$ & 0.62 \\
\hline Restaurantes y Hoteles & $2.5 \%$ & $4.1 \%$ & 0.50 & $3.4 \%$ & $4.0 \%$ & 0.67 & $3.8 \%$ & $4.4 \%$ & 0.71 \\
\hline $\begin{array}{l}\text { Transporte, Almacenamiento y } \\
\text { comunicación }\end{array}$ & $2.3 \%$ & $11.2 \%$ & 0.17 & $2.7 \%$ & $13.1 \%$ & 0.16 & $2.2 \%$ & $12.2 \%$ & 0.15 \\
\hline Intermediación Financiera & $2.0 \%$ & $2.5 \%$ & 0.63 & $2.0 \%$ & $2.9 \%$ & 0.54 & $2.8 \%$ & $2.4 \%$ & 0.95 \\
\hline Administración Pública y Defensa & $10.2 \%$ & $14.2 \%$ & 0.58 & $11.2 \%$ & $10.9 \%$ & 0.79 & $12.0 \%$ & $12.6 \%$ & 0.78 \\
\hline Enseñanza & $18.2 \%$ & $3.5 \%$ & 4.16 & $16.1 \%$ & $3.9 \%$ & 3.21 & $17.1 \%$ & $5.1 \%$ & 2.73 \\
\hline Salud Humana y Servicios Sociales & $13.5 \%$ & $4.0 \%$ & 2.72 & $9.5 \%$ & $2.9 \%$ & 2.50 & $10.1 \%$ & $4.1 \%$ & 2.03 \\
\hline Servicio doméstico & $20.5 \%$ & $0.6 \%$ & 30.11 & $21.3 \%$ & $0.6 \%$ & 29.36 & $21.8 \%$ & $0.3 \%$ & 56.90 \\
\hline $\begin{array}{l}\text { TOTAL RAMAS SELECCIONADAS } \\
(\%)\end{array}$ & $83.5 \%$ & $66.0 \%$ & 1,02 & $81.3 \%$ & $67.5 \%$ & 0,93 & $83.0 \%$ & $68.7 \%$ & 0,99 \\
\hline POB. ASALARIADA (en miles) & 2.970 & 3.677 & - & 3.610 & 4.682 & - & 3.945 & 4.818 & - \\
\hline
\end{tabular}

Fuente: Elaboración propia en base a microdatos EPH.

Referencias: \%M es el porcentaje de mujeres en cada rama respecto al total de asalariadas; \%V es el porcentaje de varones en cada rama respecto al total de asalariados; IF es el Índice de Feminización por rama

Es decir, que las ramas que generaron la disminución de la segregación horizontal medida por el índice de Duncan son: a) por un lado, aquellas con similar distribución entre sexos aunque con índices de feminización menores a uno (Ind. Textiles, confecciones y calzado, Comercio; Restaurantes y Hoteles; Intermediación Financiera; Administración Pública y Defensa). Aquí las mujeres incrementaron su participación y por ende, la feminización se acercó más la unidad; b) por el otro lado, ocupaciones típicamente femeninas donde históricamente las mujeres son mayoría (Educación; Salud Humana y Servicios Sociales). En estas ramas, creció la proporción de hombres y la feminización disminuyó, aunque aún permanece en valores entre 2 y 3 ; y c) Finalmente, las que concentran más varones o más mujeres, continuaron reforzando esa situación.

\section{Conclusiones}

El estudio de los cambios ocurridos en el mercado de trabajo durante el período 20032017 permitió detectar que las brechas de género se han reducido, pero no necesariamente como consecuencia del avance de las mujeres. En este sentido, la menor diferencia en la tasa de actividad se dio por la reducción en la participación de los varones, que fue superior al aumento registrado entre las mujeres. En cambio, en la tasa de empleo las mujeres aumentaron en mayor proporción la población ocupada que los varones, aunque este aspecto también tiene sus matices.

Por un lado, al realizar las 
descomposiciones se esas variaciones, encontramos que ante la misma propensión a participar de cada grupo, el nivel educativo (preferentemente en mujeres) favorecieron una mayor tasa de empleo en el análisis entre puntas de la serie. No obstante, las variaciones observadas por etapa tuvieron un alto componente interno en el caso de los varones. Estos resultados sugieren la posibilidad de que las mayores credenciales educativas de las mujeres podrían estar comenzando a ser valoradas.

Por otro lado, los cambios en la cantidad y calidad del empleo fueron diferentes en ambas etapas. En la primera, la tasa de empleo creció para el conjunto de la sociedad y se creó mayor proporción de trabajo asalariado registrado (principalmente varones). En ese periodo se produjo una caída significativa de la brecha salarial que podría estar fundamentada en la menor segregación horizontal y la mayor cantidad promedio de horas trabajadas por las mujeres, dado que la segregación vertical se mantuvo constante. Algunos resultados preliminares indican que en la rama Servicios Sociales y de Salud, donde han perdido participación las mujeres, se dio una mejora sustantiva en el nivel educativo de las asalariadas y en la reducción de la brecha salarial.

En la segunda etapa, y como consecuencia de las políticas macroeconómicas implementadas a partir de 2016, la tasa de empleo de las mujeres creció a una tasa más baja, siendo en su mayoría trabajo por cuenta propia. En cambio, la de los varones disminuye principalmente por puestos que eran en relación de dependencia, por ende, la proporción de autoempleo aumenta. No obstante, dentro de los asalariados la brecha de ingresos disminuye levemente, lo que podría estar explicado por la menor cantidad de varones en puestos directivos, dado que la brecha de horas semanales trabajadas y la segregación horizontal se mantienen constantes. También es posible que haya jugado un rol importante la diferencia que consigue cada rama de actividad en paritarias, habida cuenta de la composición por sexo que poseen.

En definitiva, la evolución de la cantidad y calidad de los puestos de trabajo con la consecuente reducción de las brechas se dio por lo ocurrido en la primera etapa, dado que en la segunda prevaleció el crecimiento (desacelerado) de la cantidad a costa de una peor calidad en las mujeres, y la disminución en la cantidad y calidad en los varones. Como resultado algunas brechas se estancaron y otras disminuyeron levemente. Entre las características que aún no se modifican se encuentra el nivel de concentración femenina dentro de la estructura ocupacional. Los valores alcanzados en 2017 no son muy diferentes a los que se alcanzaron en 2003 o incluso hace veinte años atrás. Todavía hay alrededor del $72 \%$ de mujeres que se concentran en las mismas cinco ramas de actividad. Lo único que se modificó es su distribución: en Comercio y Administración Pública, crecieron a un ritmo mayor que los varones, en Educación y Salud Humana y Servicios Sociales, aumentaron a una tasa menor que los varones y en Servicio Doméstico intensificaron su participación. No olvidemos que esta última es la rama de actividad con el peor nivel de ingresos, de trabajo no registrado, de nivel educativo, de estabilidad y que es considerado un trabajo no calificado.

Lo cierto es que estos resultados dejan 
abiertos una serie de interrogantes que pueden ser respondidos en futuras investigaciones. En primer lugar, aquellas que analicen en profundidad los cambios en la participación y el empleo. En segundo lugar, las que indaguen a través de modelos econométricos los determinantes de la reducción de la brecha salarial. Y por último, el eje fundamental sería conocer lo que ocurre con la distribución de los tiempos de trabajo remunerado y no remunerados. Sin esto será imposible determinar políticas públicas adecuadas para generar el logro consecutivo de un mejor nivel de bienestar social con equidad de género.

\section{Referencias bibliográficas}

Actis Di Pasquale, E. \& Atucha, A. J. (2003). Brechas salariales: discriminación o diferencias de productividad. Momento Económico, (126), 23-33.

Actis Di Pasquale, E. \& Lanari, M.E. (2015). El mercado laboral escenario de desigualdades persistentes. Las brechas abiertas de Argentina. In: M. E. Lanari y C. Hasanbegovic (comp.) Mujeres de Latinoamérica. El presente en veintidós letras (pp. 213-270). Mar del Plata: Eudem, UNMdP.

Amarante, V. y Espino, A. (2002). La evolución de la segregación laboral por sexo en Uruguay (1986-1999). Segunda Época, IX (1), 165-195.

Anker, R. (1998). Gender and Jobs: Sex segregation of occupations in the world. Genova: ILO.

Arakaki, A. y Pacífico, L. (2015, agosto). La EPH en su laberinto, viejos y nuevos desafíos. Ponencia presentada en el $12^{\circ}$ Congreso Nacional de Estudios del Trabajo, Buenos Aires, Argentina.

Beccaria, L. A., Maurizio, R. y Vázquez, G. (2017). El estancamiento de la tasa de participación económica femenina en Argentina en los 2000. Desarrollo Económico, 57 (221), 3-31.

Castillo, V., Esquivel, V., Rojo, S., Tumini, L. y Yoguel, G. (2008). Los efectos del nuevo patrón de crecimiento sobre el empleo femenino, 2003-2006. In: M. Novick, M.S. Rojo y V. Castillo (comp.) El trabajo femenino en la post convertibilidad: Argentina 2003-2007 (pp. 21-43). Sgo. de Chile: CEPAL.

Cerrutti, M. (2000). Economic reform, structural adjustment and female labor force participation in Buenos Aires, Argentina. World Development, 28(5), 879-891.

Cortés, R. (2003). Mercado de trabajo y género. El caso argentino, 1994-2002. In: M.E. Valenzuela (Ed.) Mujeres, Pobreza y Mercado de Trabajo. Argentina y Paraguay (pp. 67-103). Santiago: OIT.

Duncan O. y Duncan B. (1955). A methodological analysis of segregation indexes. American Sociological Review, 20, 210-217.

Elías, J. \& Nopo, H. (2010). The Increase in Female Labor Force Participation in Latin America 1990-2004: Decomposing the Changes. (Mimeographed document). Washington, DC: IADB. 
Flückiger, Y. and Silber, J. (1999). The Measurement of Segregation in the Labor Force. Heidelberg, New York: PhysicaVerlag.

Gasparini, L. y Marchionni, M. (2015). Overview. In: L. Gasparini y M. Marchionni (eds.). Bridging gender gaps? The rise and deceleration of female labor force participation in Latin America. La Plata: CEDLAS y IDRC.

Gasparini, L., Marchionni, M., Badaracco, N. and Serrano, J. (2015). Characterizing female participation changes. In: L. Gasparini y M. Marchionni (eds.). Bridging gender gaps? The rise and deceleration of female labor force participation in Latin America. La Plata: CEDLAS y IDRC.

MTEySS -Ministerio de Trabajo, Empleo y Seguridad Social- (2017). Las mujeres en el mundo del trabajo. Recuperado de: https://www.argentina.gob.ar/ sites/default/files/informe_ctio_ documentodetrabajo.pdf

MTEySS -Ministerio de Trabajo, Empleo y Seguridad Social- (2018). Mujeres en el mercado de trabajo argentino. Recuperado de:http://www.trabajo.gob.ar/downloads/ estadisticas/mujeres_mercado_de_ trabajo_argentino-3trim2017.pdf

Vozzi, F. y Lafuente Duarte, R. (2017, agosto). Desigualdad de género en el mercado laboral argentino actual. Ponencia presentada en el $13^{\circ}$ Congreso Nacional de Estudios del Trabajo, Buenos Aires,
Argentina.

Wainerman, C. (1996). ¿Segregación o discriminación? El mito de la igualdad de oportunidades. Boletín Informativo Techint, 285, 59-75. 